\title{
Responsiveness of urinary markers of bone resorption to orchiectomy and clodronate treatment in mature rats: a comparative study
}

\author{
Alessandro Rubinacci, Isabella Villa, Valeria Sibilia ${ }^{1}$, Carmela Netti ${ }^{1}$, Gianluigi Moro and Antonio Pecile ${ }^{1}$ \\ Unità Metabolica dell' Osso, Istituto Scientifico H San Raffaele, Via Olgettina 60, 20132 Milano, Italy and ${ }^{1}$ Dipartimento di Farmacologia, \\ Chemioterapia, Tossicologia Medica, Via Vanvitelli 32, 20129 Milano, Italy
}

(Correspondence should be addressed to A Pecile, Dipartimento di Farmacologia, Chemioterapia, Tossicologia Medica, Università di Milano, Via Vanvitelli 32, 20129 Milano, Italy)

\begin{abstract}
Objective: The aim of this study was to assess the responsiveness of two classes of bone resorption markers to the enhancement of osteoclastic activity induced by orchiectomy and to its inhibition by clodronate treatment in mature rats.

Design: Bone mineral density (BMD) at femural metaphysis, femural diaphysis, lumbar vertebrae, and the urinary excretion of pyridinoline (Pyr), deoxypyridinoline (D-Pyr), galactosylhydroxylysine (GHyl) and glucosylgalactosylhydroxylysine (GGHyl) were monitored at regular intervals for 30 days prior to and for 60 days following orchiectomy in eleven rats, divided into two groups: five rats untreated and the other six treated with clodronate.

Results: Prior to orchiectomy, a significant $(P<0.01)$ decrease in BMD was observed only at the distal femural metaphysis. This decrease appeared to be associated with a time-dependent increase in the urinary excretion of all markers. Following orchiectomy, the BMD of the untreated group decreased significantly $(P<0.01)$ at all bone sites. The bone loss was accompanied by a significant $(P<0.01)$ increase in Pyr and D-Pyr concentrations in urine, whereas urinary GHyl and GGHyl did not change significantly. In the clodronate-treated group, the BMD of the three skeletal sites did not change significantly, while the urinary excretion of all urinary biochemical markers decreased significantly $(P<0.001)$.

Conclusion: This study showed that pyridinolines are able to monitor the bone response to orchiectomy and to clodronate treatment response in androgen-deficient mature male rat, whereas glycosides appear prone to confounding factors.
\end{abstract}

European Journal of Endocrinology 138 120-127

\section{Introduction}

There is increasing interest in identifying biochemical markers of bone resorption to allow easy and frequent assessment of bone status during bone diseases and therapy. An ideal marker of bone resorption should respond appropriately to a specific block of osteoclast activity in diseases known to be associated with enhanced bone resorption, and it should also correlate with changes in bone mass as induced by either pathology or treatment (1). Validation of such biochemical markers requires a biological model that strictly controls all known factors that might affect the levels of the markers, including circadian rhythms, diet, age, gender, body and bone masses, physical activity and glomerular function. Since these factors can be controlled in the rat model, it has been used to test the new markers of bone resorption. Collagen cross links, i.e. pyridinoline (Pyr) and deoxypyridinoline (D-Pyr), have been validated in different models of osteopenia in rats induced by ovariectomy $(2,3)$, orchiectomy (4), adjuvant polyarthritis (2), calcium restriction and parathyroid hormone infusion (5). Since pyridinolines derive only from mature collagen, are released into plasma during bone collagen breakdown, and respond appropriately to the challenging conditions described above, they appear capable of fulfilling the requirements for valid markers of bone resorption in rat models of osteopenia. However, despite the acknowledged importance of comparative studies to define the potential usefulness of bone markers (1), pyridinolines have not been widely compared with other bone collagen breakdown products such as glycosides. Galactosylhydroxylysine (GHyl), that can be further glycosylated to glucosylgalactosylhydroxylysine $(\mathrm{GGHyl})$, is peculiar to collagens, is released into plasma during collagen breakdown, and does not appear to be metabolised further when collagen is degraded. GHyl has a potential use 
as a marker of bone resorption, but it has never been a subject of intense development because of the analytical complexity of the original assay (6). The newest assays are simpler, more practical (7-9), and allow a reconsideration of GHyl as a candidate marker of bone resorption.

In a previous study performed in this laboratory, cross links and glycosides excreted in urine showed different sensitivities in monitoring the bone response to ovariectomy; glycosides were not able to detect the bone sparing effect of oestrogen administration (3). To characterize further the behaviour of these markers under gonadal deficiency whilst avoiding possible interference due to effects of oestrogen on production (3), protein binding and/or renal handling of collagen catabolites (10), the urinary excretion of biochemical markers was measured in male rats during the development of osteopenia induced by surgical castration. The male orchiectomised rat was chosen as it responds to sex hormone deficiency by showing clearly enhanced bone resorption and reduced bone formation (11-13). The ovariectomised female rat shows enhancement of both resorption and formation in response to oestrogen deficiency (14). Furthermore, the reliability of these markers in reflecting specific changes in osteoclastic activity was assessed by administration of a potent inhibitor of osteoclasts, e.g. the bisphosphonate clodronate (15-17). The possible relationship between the changes in urinary excretion of biochemical markers and bone mass was determined by the simultaneous monitoring of both parameters in the same rat over time. The study was performed in adult animals to minimize the interfering effects resulting from active growth and modelling (11).

\section{Materials and methods}

\section{Animals}

Eleven male Sprague Dawley ex-breeder rats (Charles River, Calco, Italy), 8 months old at the beginning of the study were used. All the rats were housed individually at $25^{\circ} \mathrm{C}$ with a $12 \mathrm{~h}$ light $/ 12 \mathrm{~h}$ darkness cycle; a standard rat diet (Charles River, Calco, Italy) and water were available ad libitum. Orchiectomy (GDX) was performed under ether anaesthesia. On the days of urinary collection, the animals were kept in metabolic cages (Tecniplast, Varese, Italy). Twenty-four-hour urine samples were frozen immediately and stored at $-20^{\circ} \mathrm{C}$ until the measurement of urinary markers of bone resorption was performed. On each day of urinary collection the animals were weighed.

\section{Assays}

Urinary GHyl and GGHyl were measured by reversedphase chromatography. Urine samples $(500 \mu \mathrm{l})$ were diluted with $9 \mathrm{ml}$ deionised water and $1 \mathrm{ml} 100 \mathrm{mmol} / \mathrm{l}$ acetic acid and loaded onto cation exchanger solid phase extraction (SPE) columns (Bakerbond sulphonic acid, $500 \mathrm{mg}$; Baker, Deventer, The Netherlands). Samples were eluted with $1.5 \mathrm{ml}$ of a solution of $200 \mathrm{mmol} / \mathrm{l} \mathrm{NaHCO}_{3}$ and $470 \mathrm{mmol} / \mathrm{l} \mathrm{LiCl}(\mathrm{pH} \mathrm{10}$ ), then dried and reconstituted with $1 \mathrm{ml} 200 \mathrm{mmol} / \mathrm{l}$ $\mathrm{NaHCO}_{3}$ buffer ( $\mathrm{pH}$ 9.2) and derivatised with dabsyl chloride (Fluka, Buchs, Switzerland) in acetone. A $20 \mu \mathrm{l}$ aliquot of the treated sample was injected onto a C18 reverse-phase column. Separation was achieved in gradient mode at a flow rate of $0.8 \mathrm{ml} / \mathrm{min}$ and detected spectrophotometrically at $436 \mathrm{~nm}$ as previously described $(9,18)$. The detection limit was $2 \mu \mathrm{mol} / \mathrm{l}$ for both the analytes. Total interassay and intra-assay coefficients of variation were $5 \%$ and $7 \%$ respectively. The amounts of GHyl and GGHyl were expressed as nmol/24h. The methodology (9) did not use an internal standard because of the complexity of chromatograms of biological samples such as urine. When some di-amino monocarboxylic acids and analogues of hydroxylysine and lysine (diamino-propionic and -butyric acid, besides ornithine) were tested, the specific peaks could not be identified separately from peaks associated with other components in the treated urine samples. In practice, the uncertainty of analysis within-batches and betweenbatches was sufficiently low for the purposes of this study. To reduce such uncertainty further, samples from the same rat were processed in a single run.

Total urinary Pyr and D-Pyr levels were measured by reversed-phase chromatography according to the method of Eyre et al. (19). Urine samples $(250 \mu \mathrm{l})$ were hydrolysed in an equal volume of $12 \mathrm{~mol} / \mathrm{l} \mathrm{HCl}$ at $110^{\circ} \mathrm{C}$ overnight. Hydrolysates were mixed with glacial acetic acid and n-butanol and applied to the SPE CF-1 cellulose columns (Bio-Rad Laboratories, Segrate, Italy) to extract the analytes. Samples were eluted with $1.5 \mathrm{ml}$ distilled water, dried and reconstituted with $250 \mu \mathrm{l} 1 \%$ heptafluorobutyric acid. A $50 \mu \mathrm{l}$ aliquot of the sample was injected onto a $\mathrm{C} 18$ reverse-phase column. Separation was achieved isocratically and native fluorescence monitored (Ex $295 \mathrm{~nm}$; Em $395 \mathrm{~nm}$ ). The detection limit was $20 \mathrm{pmoles} / \mathrm{ml}$ for both analytes. The interassay variation was $9.3 \%$ for Pyr and $10.4 \%$ for D-Pyr. The amounts of Pyr and D-Pyr are expressed as $\mathrm{nmol} / 24 \mathrm{~h}$.

\section{Dual energy X-ray absorptiometry (DXA)}

The rats were anaesthetised with ketamine hydrochloride $(100 \mathrm{mg} / \mathrm{kg}$, i.p.) plus xylazine $(4 \mathrm{mg} / \mathrm{kg}$, i.p.) and scanned with an Hologic QDR-1000 instrument (Hologic Inc., Waltham, MA, USA) in the ultra-high resolution mode with a longitudinal line spacing of $0.254 \mathrm{~mm}$, equipped with a collimator $1.0 \mathrm{~mm}$ in diameter and with the High Resolution Software (version 4.47) analysis adapted for small animals. The femur and lumbar spine were scanned. The region of interest (ROI) was positioned 
Table 1 Bone mineral density values of three skeletal sites measured on $30\left(t_{-30}\right)$ and $15\left(t_{-15}\right)$ days before GDX and just prior to GDX $\left(\mathrm{t}_{0}\right)$.

\begin{tabular}{lccc}
\hline & \multicolumn{3}{c}{ Bone mineral density $\left(\mathrm{g} / \mathrm{cm}^{2}\right)$} \\
\cline { 2 - 4 } Skeletal site & $\mathrm{t}_{-30}$ & $\mathrm{t}_{-15}$ & $\mathrm{t}_{0}$ \\
\hline Lumbar vertebrae & $0.3068 \pm 0.0057$ & $0.3013 \pm 0.0049$ & $0.2933 \pm 0.0027$ \\
Femoral metaphysis & $0.3441 \pm 0.0037$ & $0.3283 \pm 0.0048$ & $0.3074 \pm 0.0074^{*} \dagger$ \\
Femoral diaphysis & $0.3953 \pm 0.0051$ & $0.4069 \pm 0.0053$ & $0.4082 \pm 0.0068$ \\
\hline
\end{tabular}

${ }^{*} P<0.01$ vs $_{-30} ; \dagger P<0.01$ vs $\mathrm{t}_{-15}$.

at the distal metaphysis and middle diaphysis of femur, and over four vertebrae. The software provides the total area $\left(\mathrm{cm}^{2}\right)$ of the planar image of the selected segment, bone mineral content (BMC, g) and bone mineral density (BMD, $\mathrm{g} / \mathrm{cm}^{2}$ ) of the ROI. The measurement of lumbar vertebrae of each individual animal represented the mean BMD of four lumbar vertebrae. The precision of BMD measurement by DXA was $1 \%$ whereas the linear measurement to calculate automatically the bone area did not reach that level of precision. However, the number of repeated measurements during the three months of observation was sufficient to exclude possible enlargement of bone areas.

\section{Experimental procedures}

After 14 days of adaptation to the new environment, all rats were subjected to three measurements of BMD every 14 days concomitant with $24 \mathrm{~h}$ urine collections for measurements of Pyr, D-Pyr, GHyl and GGHyl (preorchiectomy period). Thereafter, all rats were gonadectomised and divided into two groups as follows: the untreated group $(n=5)$ received vehicle $(0.9 \%$ $\mathrm{NaCl})$ whereas the treated group $(n=6)$ received clodronate twice weekly $(161 \mu \mathrm{mol} / \mathrm{l} / \mathrm{kg}$ body weight $)$ (Clasteon, Istituto Gentili, Pisa, Italy). The treatment started immediately after surgery and continued for 60 days. During the treatment period urinary collection was performed at $7,14,21,30$ and 60 days after GDX and BMD was determined at 21,30 and 60 days after GDX. As bone parameters and urinary excretion of pyridinolines and glycosides were monitored in the same animal before and after GDX, each rat being its own control, a separate sham-operated group was not used.

\section{Statistics}

Data obtained before orchiectomy were analysed by means of single factor ANOVA for repeated measurements and Duncan's multiple range test. After orchiectomy differences between groups were assessed by means of single factor ANOVA for repeated measurements with one covariate (values at $t_{0}$ ) and type of treatment as grouping factor. Interaction between treatment and time was also tested (procedure 2V-BMDP Statistical Software). Differences between values at $t_{0}$ and values at each postorchiectomy time were tested in the two groups by means of the Student's $t$-test for paired observations with Bonferroni correction for multiple comparisons. Correlation coefficients were assessed by means of the Spearman rank correlation analysis. Data are expressed as means \pm S.E.M.

\section{Results}

Prior to orchiectomy ( $t_{-30}$ to $t_{0}$ ), the BMD of lumbar vertebrae and femural diaphysis did not change, whereas the BMD of the distal femural metaphysis decreased significantly $(P<0.01$, Table 1$)$. A significant early increase of Pyr $(P<0.01)$, D-Pyr $(P<0.01)$, GHyl $(P<0.05)$ and GGHyl $(P<0.05)$ was detected in the urine samples 15 days before the BMD decrease (Table 2).

Following orchiectomy, the BMD of the untreated group decreased significantly $(P<0.01)$ at all skeletal sites, i.e. femural diaphysis, femural metaphysis, and

Table 2 Urinary excretion of D-Pyr, Pyr, GHyl and GGHyl on days $30\left(t_{-30}\right), 15\left(t_{-15}\right)$ and $0\left(t_{0}\right)$ before GDX.

\begin{tabular}{lccr}
\hline & \multicolumn{3}{c}{ Urinary excretion $(\mathrm{nmol} / 24 \mathrm{~h})$} \\
\cline { 2 - 4 } Urinary markers & \multicolumn{1}{c}{$\mathrm{t}_{-30}$} & \multicolumn{1}{c}{$\mathrm{t}_{-15}$} & \multicolumn{1}{c}{$\mathrm{t}_{0}$} \\
\hline D-Pyr & $4.72 \pm 0.89$ & $7.84 \pm 0.52^{\star *}$ & $7.88 \pm 0.72^{\star \star}$ \\
Pyr & $11.93 \pm 1.84$ & $18.73 \pm 1.51^{\star *}$ & $17.91 \pm 1.23^{\star *}$ \\
GHyl & $270.37 \pm 43.36$ & $393.03 \pm 48.56$ & $411.31 \pm 34.20^{\star}$ \\
GGHyl & $125.65 \pm 27.57$ & $206.63 \pm 17.31^{*}$ & $197.40 \pm 19.23^{*}$ \\
\hline
\end{tabular}

${ }^{*} P<0.05,{ }^{* *} P<0.01$ vs $\mathrm{t}_{-30}$. 
lumbar vertebrae. The reduction of the BMD reached statistical significance earlier at the distal femoral metaphysis $\left(t_{21}\right)$, than at lumbar vertebrae $\left(t_{30}\right)$ and femoral diaphysis $\left(\mathrm{t}_{60}\right)$ (Fig. 1 ). The BMD did not change significantly at any of the measurement sites in the group treated with clodronate. BMD changes over time between untreated and treated rats were significantly different $(P<0.01)$ at the three measurement sites. In the untreated group the bone loss was accompanied by a significant increase over time of the urinary excretion of DPyr and Pyr. Urinary excretion of GHyl and GGHyl tended to increase without reaching statistical significance. In the treated rats the urinary excretion of all biochemical markers decreased significantly to below $\mathrm{t}_{0}$ values (Fig. 2), i.e. D-Pyr $(P<0.001)$, Pyr $(P<0.001)$, GHyl $(P<0.001)$, GGHyl $(P<0.01)$. The increase of Pyr and D-Pyr was not accompanied by any change in the Pyr/D-Pyr ratio; this ratio remained constant throughout the observation time, averaging $2.56 \pm 0.08$. The excretion over time between untreated and treated groups was significantly different for all markers $(P<0.001)$.
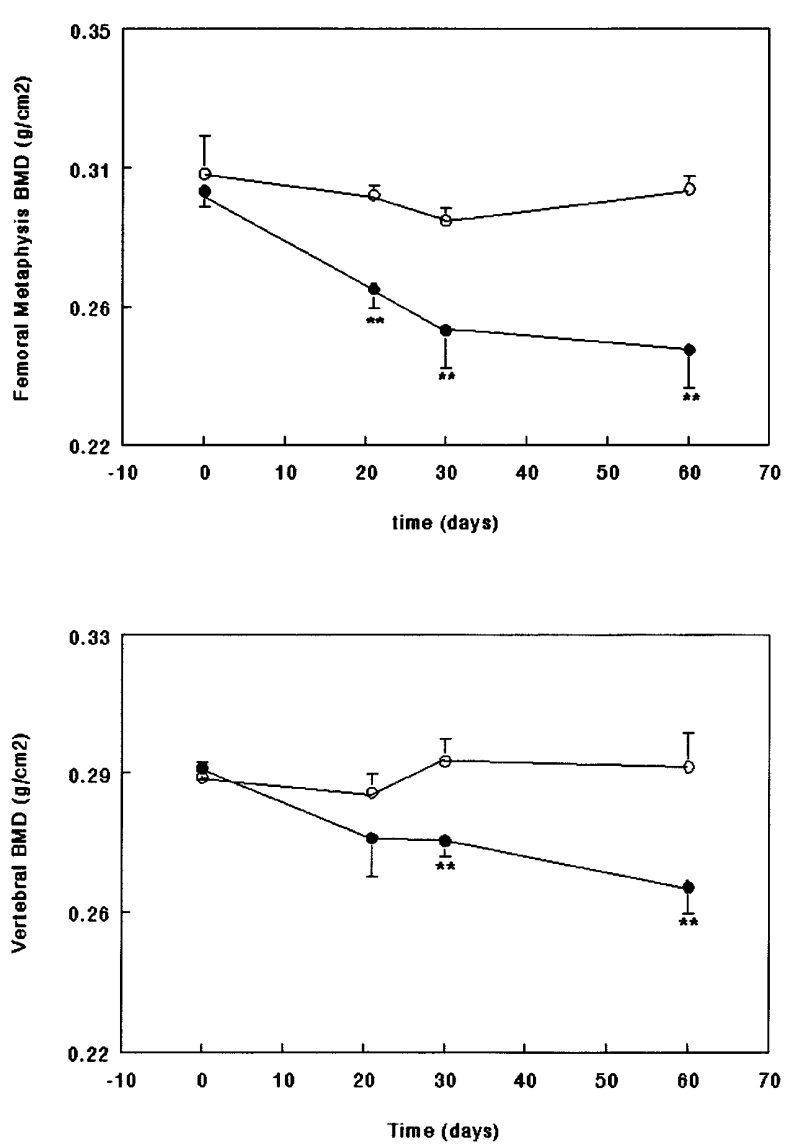

As there was a time-dependent variation in urinary excretion of the markers, the highest marker values following orchiectomy (independent from the time they were reached) and the lowest ones during clodronate treatment were considered for each rat. After orchiectomy, the mean maximum increases of D-Pyr and Pyr were statistically significant $(P<0.01)$ and nearly twofold the basal values, whereas those of GHyl and GGHyl just reached significance $(P<0.05)$. During clodronate treatment the mean maximum decreases of all markers were statistically significant. The highest mean maximum decrease was observed for D-Pyr (Fig. 3). The treatment did not significantly modify the Pyr/D-Pyr ratio; this ratio remained constant throughout the observation time, averaging $2.75 \pm 0.12$.

The femural areas of both untreated (1.941 \pm $\left.0.077 \mathrm{~cm}^{2}\right)$ and treated $\left(2.033 \pm 0.112 \mathrm{~cm}^{2}\right)$ rats did not change significantly throughout the observation time. The animals did not gain weight and their final body weights did not differ significantly in untreated $(602 \pm 40 \mathrm{~g})$ and treated $(574 \pm 30 \mathrm{~g})$ groups.

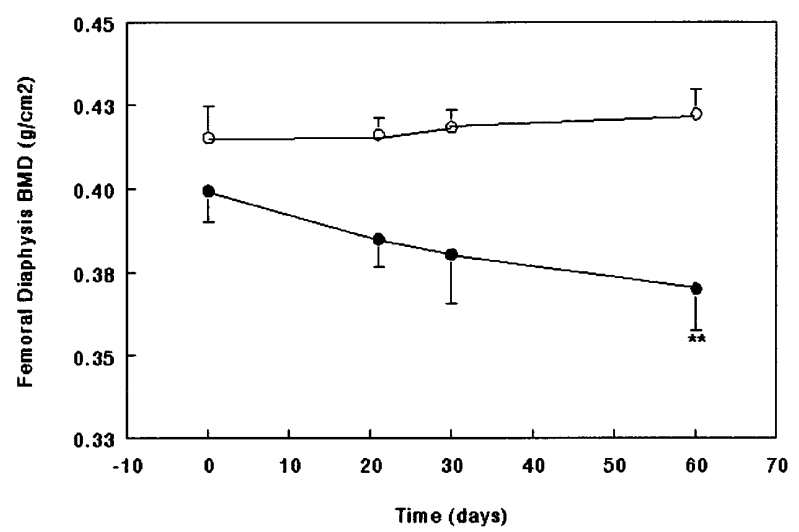

Figure 1 Bone mineral density (BMD) measured in femoral metaphysis, femoral diaphysis and lumbar vertebrae after orchiectomy (GDX). Treatment with clodronate started immediately after GDX. (๑) Untreated group $(n=5) ;(0)$ treated group $(n=6) .{ }^{* \star} P<0.01$ vs $\mathrm{t}_{0}$. 

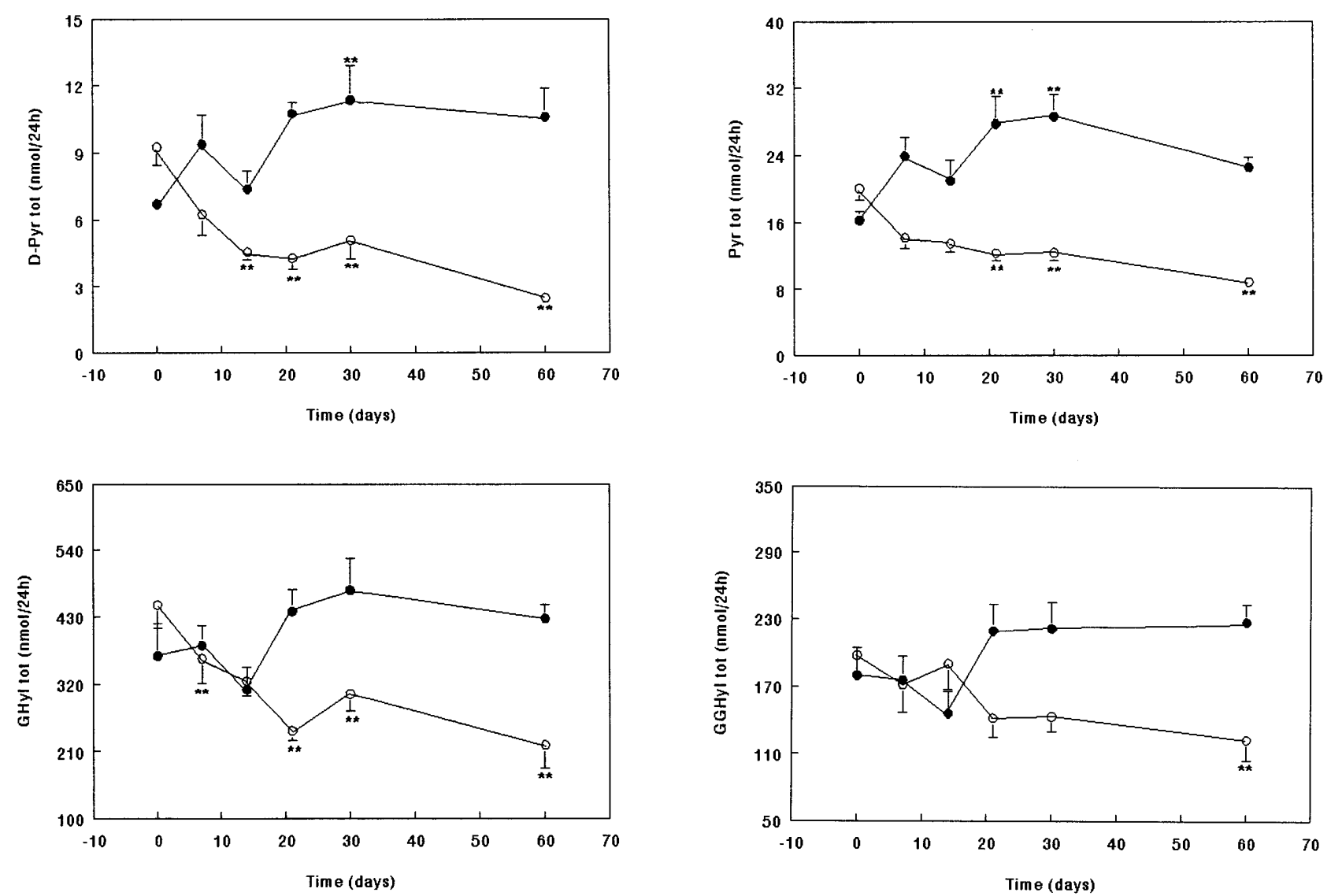

Figure 2 Urinary excretory patterns over time of D-Pyr, Pyr, GHyl, and GGHyl in untreated $(\bullet)$ and treated $(O)$ rats after orchiectomy $(G D X)$. Untreated group, $n=5$; treated group, $n=6$. ${ }^{\star \star} P<0.01$ vs $\mathrm{t}_{0}$. tot, total amount excreted in the $24 \mathrm{~h}$.

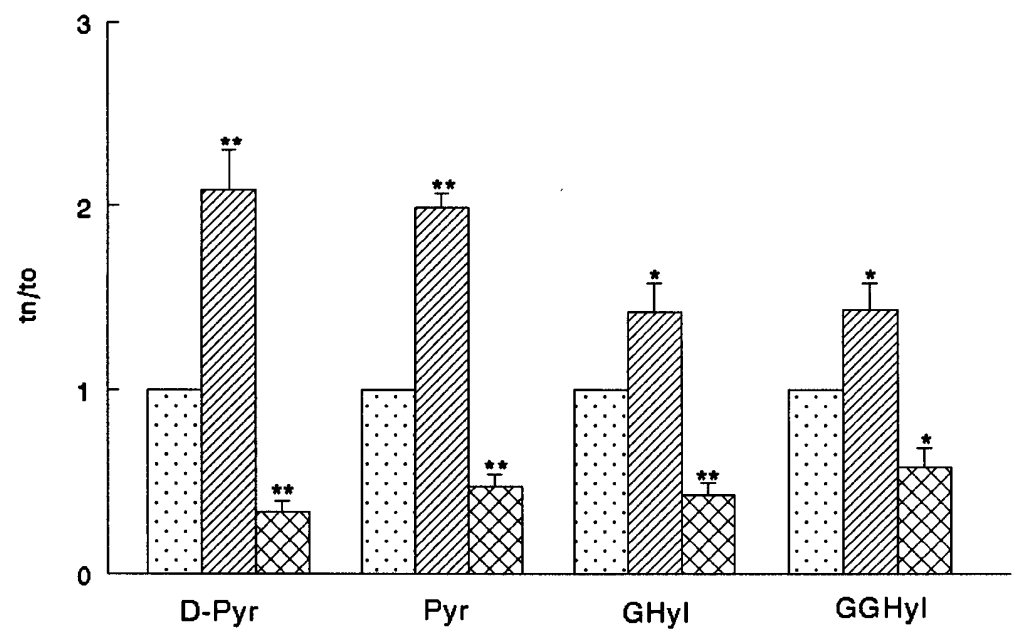

Figure 3 Maximum increase in D-Pyr, Pyr, GHyl and GGHyl expressed as a ratio of the highest urinary excretion value measured after orchiectomy $\left(t_{n}\right)$ to the urinary excretion value just before orchiectomy $\left(t_{0}\right)$ (untreated, hatched bars). Maximum decrease in D-Pyr, Pyr, GHyl and GGHyl expressed as a ratio of the lowest urinary excretion value measured during clodronate treatment $\left(t_{n}\right)$ to the basal value $\left(\mathrm{t}_{0}\right)$ (treated, cross-hatched bars). Dotted bars, basal value. ${ }^{*} P<0.05,{ }^{* *} P<0.01$ treated and untreated vs basal (Student's $t$-test for paired observations). 


\section{Discussion}

This study showed that the responsiveness of urinary pyridinium cross links and glycosides to the enhancement and subsequent block of osteoclast activity, as induced by orchiectomy and bisphosphonate treatment, were different in mature male rats. D-Pyr responded appropriately to both androgen deficiency and clodronate administration. GHyl and GGhyl detected the inhibitory effect of clodronate on bone metabolism, but were not able to monitor the bone response to orchiectomy.

Androgen deficiency induced, as expected from earlier reports $(12,13)$, a substantial loss of bone mass, but the loss was more rapid than previously shown (13), and was particularly at the expense of the cancellous compartment. The loss should be due to an increase in bone resorption rather than a decrease in bone formation since bone growth was minimal as shown by the consistency of the femural planar areas. The view that androgen-deficient mature rat loses bone because of enhanced osteoclastic activity $(11,20,21)$ is supported by previous histomorphometric data showing an increase in osteoclast number (22) and by the evidence that androgen replacement in gonadectomised rat maintains trabecular bone volume without a concomitant change in bone growth (12).

The cortical BMD loss appears to be a specific feature of male rats and might be ascribed to the porosity of the cortical wall (22), as well as to the resorption of the endosteal surface (16), thus indicating that bone remodeling space is extended to cortical bone where Haversian remodeling is normally absent. In female rats gonadal deficiency induces a loss only of the trabecular component of the bone mass whereas the cortical one remains intact (11). In addition, the bone formation rate at the periosteal surface responds to gonadal deficiency in a sex-dependent manner: it decreases in the male whereas it increases in the female rat (14).

It is worth noting that a mild bone loss was observed at the metaphyseal site even prior to orchiectomy and was found to be associated with an increase in excretion of bone collagen catabolites. Since this loss occurred when rats were eight/nine months old, it is unlikely to be related to the normal ageing process since BMD loss occurs spontaneously in males at an older age (23). Conversely, this early loss of trabecular bone mass could be due to the activation of osteoclasts following the reduction of physical activity inasmuch as the animals were breeders housed in large cages before the study and confined in smaller ones afterwards. Lack of exercise may well have had negative effects on bone mineral density (24).

This study has confirmed the bone sparing effect of clodronate by using highly sensitive methods of bone mass measurement. The present study and previous studies using scanning electron microscopy $(16,17)$ indicated that clodronate treatment prevents bone loss in adult castrated male rats.

The new findings of the present study are related to the performances of urinary excretion of four collagen breakdown products in detecting two metabolic conditions, androgen deficiency and clodronate treatment. Under these experimental conditions, urinary excretion of the four collagen breakdown products, i.e. D-Pyr, Pyr, GHyl, and GGHyl, behaved differently. D-Pyr detected the bone response to androgen deficiency, and urinary excretion decreased as a consequence of the osteoclast activity block induced by clodronate. Therefore, D-Pyr should be considered as a specific marker of bone resorption. As there is no current evidence that D-Pyr itself can be metabolically degraded (1), the larger percentage increase of D-Pyr found in the urine of male rats $(109 \%)$ after gonadectomy with respect to that $(70 \%)$ previously measured in female rats under similar experimental conditions (3), is in agreement with the greater loss of bone mass which occurred in male rats. As discussed earlier, as the bone remodeling space is extended to the Haversian system of cortical bone in male rats $(11,13)$, the greater amount of excreted D-Pyr could derive from the cortical bone, where cross linkage of bone collagen is high (25).

The decrease of the urinary excretion of D-Pyr after clodronate could be ascribed to the specific inhibition of the cell-mediated degradation of mature bone collagen. However, there is the possibility that bisphosphonate treatment affects collagen synthesis and/or maturation. It is therefore conceivable that the interpretation of the urinary excretion of cross links after chronic bisphosphonate treatment should take into account bisphosphonate-induced alterations in the composition of bone collagen cross links (5). This possibility seems to be more likely in growing animals (5) than in mature ones since, with increasing age, the synthesis of collagen decreases and the activity of lysine hydroxylase decreases as well (26). The alteration of cross-linkage in bone collagen after clodronate might be minimal in mature rats since the Pyr to D-Pyr ratio in urine samples did not change as it did in growing rats (5).

Pyr showed a less marked decrease under clodronate treatment than D-Pyr, and a similar increment after gonadectomy. The relatively low sensitivity of Pyr to bisphosphonate treatment fits with analogous observations in humans (10) and suggests that some urinary Pyr might derive from soft tissue degradation. However, soft tissue sources do not appear to interfere significantly with Pyr responsiveness to orchiectomy. In fact the ratio Pyr/D-Pyr remained constant, as it did in other bone hyperresorptive states such as those induced by oestrogen deficiency (27) and parathyroid hormone infusion (28). It is therefore possible that androgen deficiency does not activate extraosseous collagen breakdown leading to the enhancement of urinary Pyr. In contrast with the results obtained for excretion of cross links, glycosides were not able to detect the bone 
response to orchiectomy in mature rats. The lack of correlation between pyridinolines and glycosides responsiveness is unclear. The relative insensitivity of glycosides to androgen deficiency might be explained by the reported reduction in bone formation after orchiectomy. Given that glycosides could be derived from intracellular degradation of newly synthetised collagen (29), the reduction of this metabolic pathway induced by androgen deficiency could have masked the increase in urinary glycosides deriving from bone resorption.

The urinary daily output of both glycosides was greatly reduced during clodronate treatment. The reduction reached a similar degree as that shown for the pyridinolines and reflected the steady BMD at trabecular and cortical sites. It can, therefore, be argued that glycosides should have detected the osteoclast activity block induced by the bisphosphonate treatment. In addition, there is the possibility that the decrease of both glycosides could be ascribed, at least in part, to clodronate inhibition of intracellular degradation of newly synthetised collagen (30) since GGHyl and GHyl might also derive from osteoblast synthetic activity and osteoblast intracellular degradation (3, $29,31)$. Although rat bone contains more GGHyl than GHyl (32), the parallel changes of urinary glycosides are not unexpected since rat kidney cortex displays a specific $\alpha$-glucosidase which converts the glucosyl fraction into the galactosyl form (33). However, we cannot exclude the possibilty that the reduction in urinary glycosides induced by clodronate might also be due to bisphosphonate effects on extraosseous sources of GGHyl e.g. the Clq component of the complement, as clodronate is known to have anti-inflammatory and anti-arthritic actions (15). On the basis of these considerations, the changes in excretion of glycosides during clodronate treatment may reflect not only true modifications of bone resorption but also alterations induced by the bisphosphonate in the degradative metabolism of collagen and/or proteins containing collagen-like sequences.

In conclusion this longitudinal and comparative study has demonstrated that urinary excretion of pyridinolines can detect the bone response to androgen deficiency and clodronate administration whereas urinary excretion of glycosides may be affected by factors other than bone resorption.

\section{References}

1 Calvo MS, Eyre DR \& Gundberg M. Molecular basis and clinical application of biological markers of bone turnover. Endocrine Reviews 199617 333-368.

2 Tordjman C, Lhumeau A, Pastoreau P, Meunier F, Serkiz B, Volland JP et al. Evaluation and comparison of urinary pyridinium cross-links in two rat models of bone loss - ovariectomy and adjuvant polyarthritis - using a new automated HPLC method. Bone and Mineral 199426 155-167.

3 Pecile A, Sibilia V, Netti C, Villa I, Calori G, Tenni R et al. Comparison between urinary pyridinium crosslinks and hydroxylisine glycosides in monitoring the bone response to ovariectomy and $17 \beta$-estradiol replacement in aged rats. Journal of Endocrinology $1996150383-390$.

4 Vanderschueren D, Jans I, Van Herk E, Moermans K, Verhaeghe J \& Bouillon R. Time-related increase of biochemical markers of bone turnover in androgen-deficient male rats. Bone and Mineral $199426123-131$.

5 Egger CD, Mühlbauer RC, Felix R, Delmas PD, Marks SC \& Fleisch $\mathrm{H}$. Evaluation of urinary pyridinium crosslink excretion as a marker of bone resorption in the rat. Journal of Bone and Mineral Research 19949 1211-1219.

6 Krane SM, Kantrowitz FG, Byre M, Rinnel SR \& Singer FR. Urinary excretion of hydroxylysine and its glycosides as an index of collagen degradation. Journal of Clinical Investigation 197759 819-827.

7 Moro L, Battista S, Modricky C, Rovis L \& De Bernard B. Highperformance liquid chromatography preparation of galactosylhydroxylysine, a specific bone collagen marker. Journal of Chromatography 1989490 285-292.

8 Yoshihara K, Mochidome N, Shida Y, Hayakaw Y \& Nagata M. Pre-column derivatization and its optimum conditions for quantitative determination of urinary hydroxylysine glycosides by high-performance liquid chromatography. Biological and Pharmacological Bulletins 199316 604-607.

9 Grazioli V, Casari E, Murone M \& Bonini PA. High-performance liquid chromatographic method for measuring hydroxylysine glycosides and their ratio in urine as a possible marker of human bone collagen breakdown. Journal of Chromatography 1993615 59-66.

10 Garnero P, Shih WJ, Gineyts E, Karpf DB \& Delmas PD. Comparison of new biochemical markers of bone turnover in late postmenopausal osteoporotic women in response to alendronate treatment. Journal of Clinical Endocrinology and Metabolism 199479 1693-1700.

11 Kimmel DB. Animal models for in vivo experimentation in osteoporosis research. In Osteoporosis, pp 671-690. Eds R Marcus, D Feldman \& J Kelsey. San Diego: Academic Press, 1996.

12 Vanderschueren D, Van Herk E, Suiker AMH, Visser WJ, Schot LPC \& Bouillon R. Bone and mineral metabolism in aged male rats: short and long term effects of androgen deficiency. Endocrinology $19921302906-2916$.

13 Vanderschueren D, Van Herk E, Schot P, Rush E, Einhorn T, Geusen $\mathrm{P}$ et al. The aged male rat as a model for human osteoporosis: evaluation by nondestructive measurements and biochemical testing. Calcified Tissue International 199353 342-347.

14 Turner RT \& Hammnon KS. Differential effects of gonadal function in bone histomorphometry in male and female rats. Journal of Bone and Mineral Research 19894 557-563.

15 Plosker GL \& Goa KL. Clodronate: a review of its pharmacological properties and therapeutic efficacy in resorptive bone disease. Drugs 199447 945-982.

16 Wink CS, St Onge M \& Parker B. The effect of dichloromethylene bisphosphonate in osteoporotic femora in adult castrated male rats. Acta Anatomica 1985124 117-121.

17 Wink CS. A scanning electron microscopic study of femoral bone surface from castrated rats treated with dichloromethylene bisphosphonate. Acta Anatomica 1986126 57-62.

18 Alfano M, Casari E, Stenico A, Murone M \& Grazioli V. Improved HPLC method for the determination of urinary hydroxylysine glycosides to study bone turnover rate in humans and rats. Clinical Chemistry $1994402113-2114$.

19 Eyre DR, Koob TJ \& Van Ness KP. Quantification of hydroxypyridinium cross-links in collagen by HPLC. Analytical Biochemistry $1984137380-388$.

20 Schoutens A, Verhas M, L'Hermite-Baleriaux M, L'Hermite M, Verschaeren A, Dourov N et al. Growth and bone haemodynamic responses to castration in male rats. Reversibility by testosterone. Acta Endocrinologica 1984107 428-432.

21 Vanderschueren D \& Bouillon R. Androgens and bone. Calcified Tissue International $199556341-346$. 
22 Verhas M, Schoutens A, L'Hermite-Baleriaux M, Dourov N, Verschaeren A, Mone $\mathrm{M}$ et al. The effect of orchiectomy on bone metabolism in aging rats. Calcified Tissue International 198639 $74-77$.

23 Schapira D, Lotan-Miller R, Barzilai D \& Silbermann M. The rat as a model for studies of the aging skeleton. Cells and Materials 1991 Suppl 1 181-188.

24 Tuukkanen J, Peng Z \& Väänänen HK. Effect of running exercise on the bone loss induced by orchiectomy in the rat. Calcified Tissue International $19945533-37$.

25 Noris Suarez K, Romanello M, Bettica P \& Moro L. Collagen type I of rat cortical and trabecular bone differs in the extent of posttranslational modifications. Calcified Tissue International 1996 58 65-69.

26 Guenther HL, Guenther HE \& Fleisch HA. The influence of 1-hydroxyethane-1,1-diphosphonate and dichloromethylenebisphosphonate on lysine hydroxylation and cross-link formation in rat bone, cartilage and skin collagen. Biochemical Journal 1981 $196303-310$

27 Black D, Farquharson C \& Robins SP. Excretion of pyridinium cross-links of collagen in ovariectomized rats as urinary markers for increased bone resorption. Calcified Tissue International 1989 44 343-347

28 Jerome CP, Colwell A, Eastell R, Russell RGG \& Trechsel U. The effect of parathyroid hormone (1-34) infusion on urinary 3-hydroxypyridinium cross-link excretion in the rat. Bone and Mineral 199219 117-125.

29 Jilka RL \& Hamilton JW. Evidence for two pathways for stimulation of collagenolysis in bone. Calcified Tissue International 198537 300-306.

30 Gallagher JA, Guenther HL \& Fleisch HA. Rapid intracellular degradation of newly synthesized collagen by bone cells: effect of dichloromethylenebisphosphonate. Biochimica et Biophysica Acta $1982719349-355$.

31 Kucharz EJ. Biosynthesis of collagen. In The Collagens: Biochemistry and Pathophysiology, pp 31-53. Ed EJ Kucharz. Berlin: Springer Verlag, 1992.

32 Michalsky M, Norris-Suarez K, Bettica P, Pecile A \& Moro L. Rat cortical and trabecular bone collagen glycosylation are differently influenced by ovariectomy. Biochemical and Biophysical Research Communications 1993192 1281-1288.

33 Sternberg M \& Shapiro RG. Studies on the catabolism of the hydroxylysine-linked disaccharide units of basement membrane and collagens. Isolation and characterization of a rat $\alpha$-glucosidase of high specificity. Journal of Biological Chemistry 1979254 10329-10336.

Received 23 April 1997

Accepted 26 August 1997 